\title{
EXPERIMENTAL DETERMINATION OF LIDAR OVERLAP PROFILE BASED ON DUAL FIELD-OF-VIEW HIGH SPECTRAL RESOLUTION LIDAR
}

\author{
Nanchao Wang ${ }^{1}$, Xue Shen ${ }^{1}$, Yudi Zhou ${ }^{1}$, Chong Liu ${ }^{1}$, Yupeng Zhang ${ }^{1}$, Ju Ke ${ }^{1}$, Da Xiao ${ }^{1}$, Tianfen \\ Zhong ${ }^{1}$, Yuhang Rong ${ }^{1}$, Binyu Wang ${ }^{1}$, Jie Chen ${ }^{1}$ and Dong Liu ${ }^{1, *}$ \\ ${ }^{1}$ State key Laboratory of Modern Optical Instrumentation, College of Optical and Engineering, Zhejiang \\ University, Hangzhou, Zhejiang 310027, China \\ *Email: liudongopt@zju.edu.cn
}

\begin{abstract}
This paper presents two approaches to calibrate the overlap factor under inhomogeneous atmospheric condition without critical assumption and delivers detailed analysis about the retrieval errors of overlap profile in High-SpectralResolution-Lidar (HSRL). The first method employs an additional optical subsystem with different field-of-view, that is dual field-of-view HSRL, for the retrieval of overlap profile. The second method takes advantage of the difference of the result between the HSRL and Klett method, that is about the retrieval of backscatter coefficient for uncorrected lidar signal, to correct overlap profile. Surprisingly, two methods show very high-level consistency and stability of the result. It is potential that this technique would be an excellent solution for experimental determination of lidar overlap in ground-based HSRL.
\end{abstract}

\section{INTRODUCTION}

In general, literature has reported various methods to correct the overlap profile, which could be grouped into two categories: theoretical and experimental approaches. The calculations of theoretical approaches are based on the specifications of the Lidar system as the divergence angle of laser beam, the direction of laser beam and the field-of-view of the receiver, etc. The theoretical approaches could indicate what effect the configuration of Lidar system change will have on the overlap profile, and have directive meaning for designing Lidar system. The inconvenient of such approaches is that they require a good knowledge of specification to derive an overlap profile with enough accuracy, which is quite difficult to measure in the general case. The experimental determination of the overlap profile is required to derive an accurate correction with few priori knowledge. Sasano et al.
[1] proposed a simple method to derive overlap profile assuming homogeneous atmospheric condition. Also, Tomine et al. ${ }^{[2]}$ presented a method under light fog and mist condition. An approach was reported by Dho et al. ${ }^{[3]}$, that could be applied in inhomogeneous condition, based on a polynomial fitting. An enlightening approach widely used in Raman lidar about experimental determination of lidar overlap profile was reported by U. Wandinger ${ }^{[4]}$, and without critical assumption except lidar ratio. And on this basis, J. Luis et al. ${ }^{[5]}$ compared this method with the measurement of overlap profile with additional $910 \mathrm{~nm}$ ceiliometer in Ramen lidar, which is similar to our idea.

As a summary of previous work, either system specifications, atmospheric conditions, or lidar ratios are required for estimation of the overlap profile. Additional assumption inevitably leads to uncertainties in the results. And for all we know, there is limited research for experimental determination of lidar overlap without assumption in the HSRL. In this paper, we present two simple method for experimental determination of overlap profile for dual-field-of-view HSRL with no prior assumption.

\section{INSTRUMENTATION}

A highly robust dual-field-of-view HSRL that provides measurement of aerosol optical properties at $532 \mathrm{~nm}$ has been developed, tested, and deployed on field experiments by Zhejiang University. And it provides the ability to measure aerosol backscatter, extinction coefficient and depolarization.

The main system is based on Q-switched Nd:YAG laser seeded by DBR laser with fundamental emission at $1064 \mathrm{~nm}$, and additional emissions at $532 \mathrm{~nm}$ by using second harmonic generators is employed. The receiving system 
mainly consists of a $280 \mathrm{~mm}$ diameter Cassegrain telescope with a full field-of-view of $0.36 \mathrm{mrad}$, an interferential filter, a polarization cube and a beam splitter, that discriminates three detecting channels corresponding to parallel channel, perpendicular channel and molecular channel. The separation distance between transmitting and telescope is $180 \mathrm{~mm}$. The backscatter signal of molecular channel is directed to the iodine filter module which contains the iodine vapor filter and temperature controller. And it could suppress the aerosol backscatter signal and transmit most of molecular backscatter signal, that is why it is called molecular channel. The lidar backscatter signal is registered with a vertical resolution of $7.5 \mathrm{~m}$ and temporal resolution of 2-minute within $0.5-8 \mathrm{~km}$ detecting range.

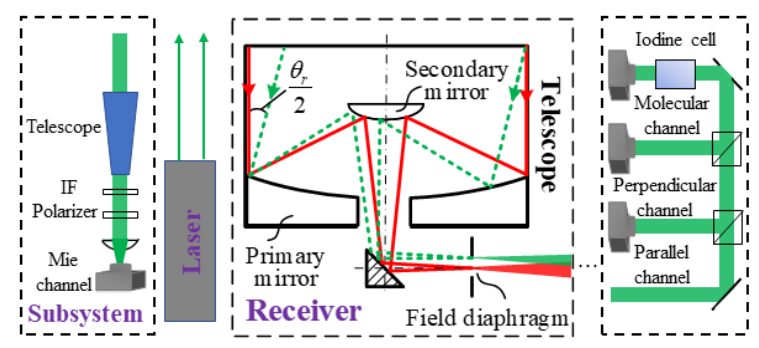

Fig. 1 The system layout of dual field-of-view HSRL system.

An additional subsystem is designed for experimental determination of overlap profile. It receives backscatter signal with different field-ofview, which is compared to main Lidar in order to retrieve overlap profile. Lidar subsystem mainly consists of a small telescope with large field-ofview, a polarizer, an interferential filter, and a photomultiplier detector which is the fourth channel called Mie channel. The detailed technique would be discussed in Section 3.

\section{METHODOLOGY}

The fundamental idea for experimental determination of overlap profile that motivates our approach is to compare two backscatter signals generated from same area with different field-ofview, that is, the deviation between two signals affected by incomplete overlap allows for retrieving the lidar overlap function. The most of experimental approaches require specific assumptions such as homogeneous atmospheric conditions or lidar ratio. Whereas our method does not need any assumption and could retrieve overlap profile real-time by taking advantage of a compact low-cost optical sub-system or an iterative approach. In section.4, we could see that the results of two method are in accordance with each other. The following paragraphs describe in detail the proposed procedure.

For the purpose of increasing the signal-toratio, it is convenient to let the polarization of subsystem parallel to the parallel channel of main system. The backscatter signal in all four channels is listed below: $P_{M i e}$ is the channel of subsystem through the polarizer, $P_{m}$ is the molecular channel filtered by iodine cell, $P_{/ /}$and $P_{\perp}$ are the parallel and perpendicular channels, respectively.

\subsection{Dual field-of-view method}

We consider the backscatter signal of subsystem as the reference profile. The measurement of subsystem is unaffected by incomplete overlap already from above the blind zone $\left(O_{1}(\mathrm{r})=0\right)$. Note here and reflected in Eq. (1) and (4), the only difference between $P_{\text {Mie }}$ and $P_{\|}$is the overlap terms due to different field-of-view of two system. It could be seen as an approximation that $\mathrm{O}_{2}(\mathrm{r}) \approx 1$ in near-filed if the filed-of-view of subsystem receiver is large enough. Then uniform $O_{1}(\mathrm{r})$ could be easily derived from the Eq. (4) by applying Eq. (6) and used to correct other channels as follow

$$
O_{1}(\mathrm{r}) \propto P_{\|}(\mathrm{r}) / P_{\text {Mie }}(\mathrm{r})
$$

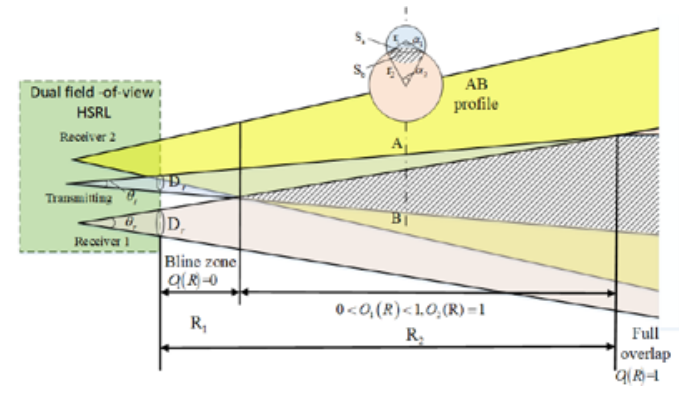

Fig.2 The schematic view of dual field-of-view.

\subsection{Iterative method}

The basic idea underlying the iterative method is that HSRL could retrieve backscatter coefficient 
without knowing the overlap profile and the backscatter coefficient retrieving by Klett method is affected by the incomplete overlap.

The difference could be used to correct overlap profile as following:

$$
\frac{\beta_{H S R L}-\beta_{\text {Klett }}}{\beta_{H S R L}+\beta_{m}} \propto \frac{P(\mathrm{r}) \cdot O^{-1}(\mathrm{z})-P(\mathrm{r})}{P(\mathrm{r}) \cdot O^{-1}(\mathrm{z})}=1-O(\mathrm{z})
$$

However, Klett method can often be unstable to retrieve overlap profile by simply applying Eq. (14). We will try to use the relative difference to reduce iteratively the incomplete overlap by correcting the backscatter signal $P(\mathrm{r})$. In next step $(\mathrm{i}+1)$, the corrected backscatter signal is used to retrieve the aerosol backscatter profile again.

$$
\begin{gathered}
\Delta O^{(\mathrm{i})}(\mathrm{r})=\frac{\beta_{H S R L}(\mathrm{r})-\beta_{\text {Klett }}^{(\mathrm{i})}(\mathrm{r})}{\beta_{H S R L}(\mathrm{r})+\beta_{m}(\mathrm{r})} \\
P^{(\mathrm{i}+1)}(\mathrm{r})=P^{(\mathrm{i})}(\mathrm{r}) \cdot\left[1+\Delta O^{(\mathrm{i})}(\mathrm{r})\right]
\end{gathered}
$$

After few iterations (about 10-20 times), this procedure could correct the effect of incomplete overlap completely. The overlap profile could be calculated as:

$$
O(\mathrm{r})=\mathrm{P}^{(1)}(\mathrm{r}) / \mathrm{P}^{(\text {end })}(\mathrm{r})
$$

\section{RESULTS}

Data used in this paper were measured at Yuquan Campus, Zhejiang University, Hangzhou, Zhejiang, China ( N30 16', E12007'07" ) during the CALIOL (Cloud-Aerosol Lidar Observation Laboratory) field campaign. The measurement of $11^{\text {th }}$ Mar 2019 took place from 00:44 until 03:29 UTC+8.

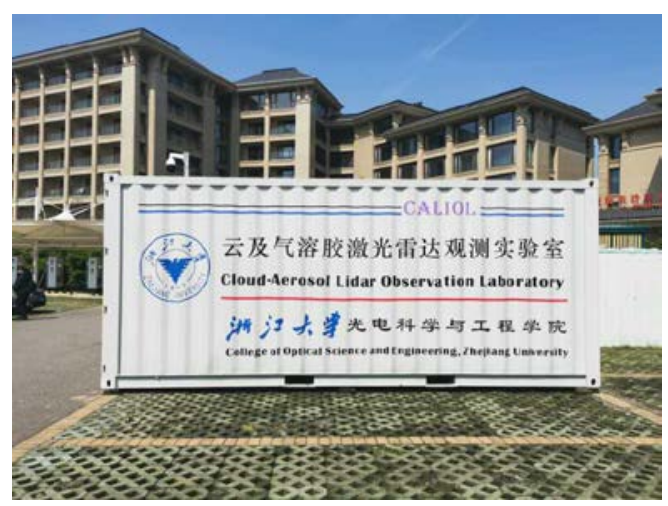

Fig.3 the field campaign of CALIOL.

Figure 4(a) presents an example of the backscatter signals obtained from parallel channel and Mie channel. The comparison of two profile shows that they are very similar above the area of full overlap. And we could easily to calculate the overlap profile.

The result of iterative method used to correct backscatter signal is also shown in Figure 4(b) for the measurement of same time. The consequence of corrected backscatter signal for each step is also illustrated. The value of lidar ratio is assumed as 40 according to the above result in the retrieval of Klett method, which is typical value of urban aerosols.
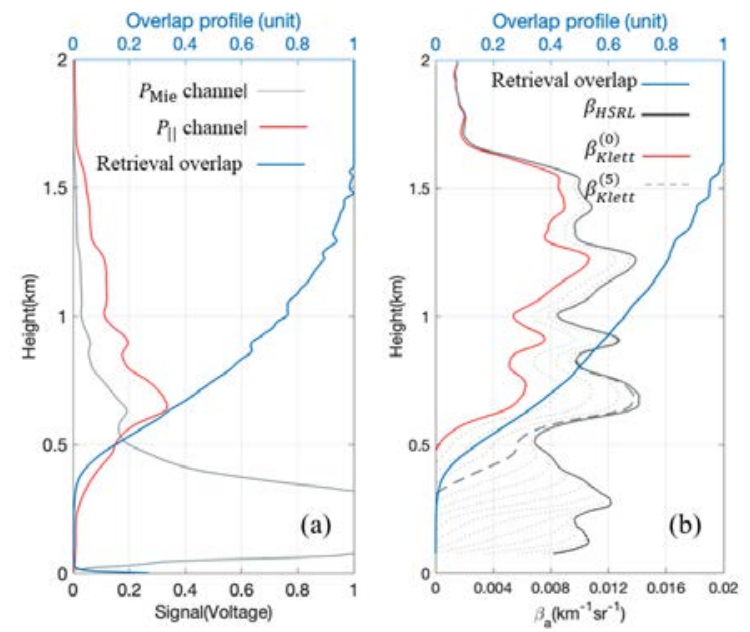

Fig.4 Overlap profile derived from dual field-ofview (a) and iterative method (b).

We take into account all 1211 profiles measured from 0:44 until 3:29 to calculate the overlap profile with two method, and the results is shown in Figure 5. The error bars (three standard deviation) show the variability of the overlap profile. It seems like that iterative method is more stable than dual field-of-view method. But the average overlap profiles of two method are almost entirely the same. Then two average overlap profiles fitting by exponential model ${ }^{[5]}$, both correlation index $\mathrm{R}^{2}>0.99$, indicating that the high-level consistency of two methods had proved the certain dependability of the results. 

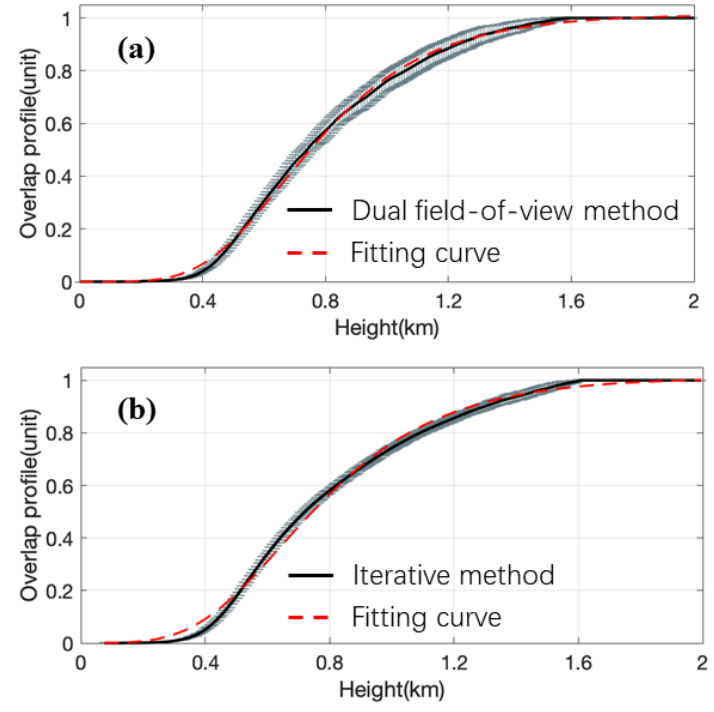

Fig.5 The results of dual field-of-view method (a) and iterative method (b).

\section{ACKNOWLEDGEMENTS}

This work was supported by the National key Research and Development Program of China (2016YFC0200704).

\section{REFERENCES}

[1] SASANO Y, SHIMIZU H, TAKEUCHI N, et al. Geometrical form factor in the laser radar equation: an experimental determination [J]. Applied Optics, 1979, 18(23): 3908-10.

[2] TOMINE K, HIRAYAMA C, MICHIMOTO $\mathrm{K}$, et al. Experimental determination of the crossover function in the laser radar equation for days with a light mist [J]. Applied Optics, 1989, 28(12): 2194-5.

[3] DHO S W, PARK Y J, KONG H J. Experimental determination of a geometric form factor in a lidar equation for an inhomogeneous atmosphere [J]. Applied Optics, 1997, 36(24): 6009-10.

[4] WANDINGER U, ANSMANN A. Experimental determination of the lidar overlap profile with Raman lidar [J]. Applied Optics, 2002, 41(3): 511. [5] JUAN LUIS $G$ R, MARIA JO?O C, DANIELE B, et al. Infrared lidar overlap function: an experimental determination [J]. Optics Express, 2010, 18(19): 20350-9 